\title{
Etude sur les acides fulviques extraits d'eaux superficielles françaises - Extraction, caractérisation et réactivité avec le chlore
}

\section{Study of aquatic fulvic acids isolated from french surface waters - Isolation procedure, characterization, and reactivity with chlorine}

\author{
B. Legube, F. Xiong, J. P. Croue et M. Doré
}

Volume 3, numéro 4, 1990

URI : https://id.erudit.org/iderudit/705082ar

DOI : https://doi.org/10.7202/705082ar

Aller au sommaire du numéro

Éditeur(s)

Université du Québec - INRS-Eau, Terre et Environnement (INRS-ETE)

ISSN

0992-7158 (imprimé)

1718-8598 (numérique)

Découvrir la revue

Citer cet article

Legube, B., Xiong, F., Croue, J. P. \& Doré, M. (1990). Etude sur les acides fulviques extraits d'eaux superficielles françaises - Extraction, caractérisation et réactivité avec le chlore. Revue des sciences de l'eau / Journal of Water Science, 3(4), 399-424. https://doi.org/10.7202/705082ar

\section{Résumé de l'article}

Après avoir présenté quelques données bibliographiques sur les substances humiques aquatiques, l'extraction de plusieurs substances humiques issues de onze eaux de surface françaises, est discutée en terme de rendements d'extraction. Quelques paramètres de caractérisation des acides fulviques sont présentés et en particulier, l'analyse élémentaire, l'absorption UV, les fonctions carboxyles et les potentiels de réactivité avec le chlore. Concernant la chloration, une attention particulière a été portée d'une part sur la nature produits organo-chlorés formés et d'autre part sur les corrélations qui existent entre la nature des acides fulviques et leur potentiel de réactivité avec le chlore. 


\title{
Étude sur les acides fulviques extraits d'eaux superficielles françaises
}

Extraction, caractérisation et réactivité avec le chlore

\author{
Study of aquatic fulvic acids isolated \\ from french surface waters \\ Isolation procedure, characterization, and reactivity with chlorine
}

B. LEGUBE, F. XIONG, J.-P. CROUE, M. DORÉ1

RÉSUMÉ

Après avoir présenté quelques données blbliographiques sur les substances humiques aquatiques, I'extraction de plusieurs substances humiques Issues de onze eaux de surface françaises, est discutée en terme de rendements d'extraction. Quelques paramètres de caractérisation des acides fulviques sont présentés et en particulier, I'analyse élémentaire, I'absorption UV, les fonctions carboxyles et les potentiels de réactivité avec le chlore. Concernant la chloration, une attention particulière a été portée d'une part sur la nature produits organo-chlorés formés et d'autre part sur les corrélations qui existent entre la nature des acides fulviques et leur potentiel de réactivité avec fe chlore.

Mots clés : substances humiques aquatiques, acides fulviques, extraction, caracterisation, chloration, produits organo-chlorés.

\section{SUMMARY}

Numerous procedure for the isolation of aquatic humic substances are described In literature. The first part of this study presents the extraction of fulvic and humic acids from eleven french surface waters, using XAD 8 macroreticular resin as developped by THURMAN and MALCOLM (1981). The results are discussed in terms of extraction yield and fulvic acid/humic acid mass ratio.

The second part of the paper presents some structural parameters of the extracted fulvic acids such as elemental analysis, UV-absorbance, carboxylefunction content and in some cases of fulvic acids, infrared spectrum and apparent molecular weight investigated by ultra filtration.

The third part of the work concerns the study of fulvic acids chlorination. Firstly, the gas chromatography - mass spectrometry analysis of one chlorinated fulvic acid allowed us to identify some chlorination by-products and especially chloroform, dichloro-acetic and trichloroacetic acids and some other chlori-

1. Laboratoire Chimie de f'Eau et des Nuisances, Ecole Supérieure d'Ingénieurs de Poitiers, Université de Poitiers, 40 avenue du Recteur Pineau, 86022 Poitiers cedex, France. 
nated allphatic acids. Secondly, the chlorine reactivity potentials of each fulvic acids were determined and results are discussed in terms of chlorine consumption, chloroform and total organohalides formation potentials. Some relationships are established between the chlorine reactivity potentials and UV-absorbance of the extracted fulvic acids.

Key-words : aquatic humic substances, fulvic acids, isolation, characterisation, chlorination, organohalides.

\section{INTRODUCTION}

Dans les eaux superficielles, environ $90 \%$ du carbone organique total est sous forme dissoute. Les substances humiques, composés organiques naturels macromoléculaires issues de la polymérisation de composés liés aux processus de dégradation biologique et d'oxydation chimique de déchets végétaux et animaux, représentent en moyenne 30 à $50 \%$ du carbone organique dissous (THURMAN, 1985).

Les matières humiques sont généralement définies par rapport à la technique d'extraction utilisée. Parmi les différents procédés retenus, l'utilisation de résines macroporeuses adsorbantes de type XAD semble le procédé le plus intéressant. Les substances sont alors définies comme la fraction organique qui s'adsorbe sur la résine XAD à pH acide (les acides organiques non retenus sont appelés acides hydrophiles (LEENHER, 1981)). La méthode établie par THURMAN et MALCOLM (1981) qui s'appuie sur cette technique est aujourd'hui proposée par l'IHSS International Humic Susbtance Society (MAC CARTY et al., 1986).

Les substances humiques sont divisées arbitrairement en deux entités: les acides humiques qui précipitent en milieu acide $(\mathrm{pH}=1$ à une concentration de $500 \mathrm{mg} \mathrm{t}^{-1} \mathrm{C}$ ) et les acides fulviques solubles à $\mathrm{pH}$ acide (THURMAN et MALCOLM, 1981). Les acides fulviques représentent toujours la fraction la plus importante (THURMAN, 1985).

La composition élémentaire moyenne des substances humiques est de 40 à $60 \%$ carbone, 4 à $6 \%$ d'hydrogène, 0,5 à $2 \%$ d'azote, 30 à $50 \%$ d'oxygène, moins de $1 \%$ de soufre et de phosphore et jusqu'à $10 \%$ de résidu (CROUE, 1987). Elle est fonction du milieu aquatique mais aussi des saisons (VISSER, 1983).

Les masses moléculaires apparentes des substances humiques déduites des procédés d'ultrafiltration et de perméation sur gel sont généralement inférieures à 10000 Daltons (GJESSING et LEE, 1967 ; AIKEN, 1984 ; THURMAN, 1985 ; COLLINS et al., 1986). Par diffraction aux rayons $X$, THURMAN ot MALCOLM (1983) ont montré que la masse moléculaire des acides fulviques 500 à 2000 Daltons est généralement plus faible que celle des acides humiques 2000 à 5000 Daltons. 
L'oxygène, principal élément après le carbone est intégré le plus souvent au sein de groupements carboxyles (4 à $6,8 \mathrm{mg} / \mathrm{g} \mathrm{SH}$ ) carbonyles et hydroxyles $(0,7$ à 3,8 meq $\mathrm{OH}$ alcools et composés phénoliques/g $\mathrm{SH})$ (OLIVER et THURMAN, 1983 ; THURMAN, 1985) ; groupements qui conditionnent les propriétés chimiques des matières humiques.

Les substances humiques incluent dans leur structure des sites aliphatiques et aromatiques. La partie aliphatique, majoritaire, reste très mal caractérisée. Elle engloberait vraisemblablement des composés comme des protéines, des acides aminés, des sucres (THURMAN, 1985 ; BRUCHET, 1985 ; NORWOOD, 1988).

Les sites aromatiques peuvent représenter jusqu'à $30 \%$ du carbone organique (THURMAN, 1985). Ils sont représentés par des noyaux aromatiques simples (substitués par des chaînes alkyles et des groupements carboxyles, cétones et hydroxyles) et des structures polycycliques (polyaromatiques et composés de type furane et pyridine) (LIAO et al., 1982 ; SCHULTEN et al., 1987).

Les principaux modèles de structure de substances humiques proposés ne représentent que leur partie aromatique. C'est le cas du modèle établi par CHRISTMAN et al. (1987), intitulé “ Aromatic Matrix \#, qui s'inspire des résultats de LIAO et al. (1982) sur la dégradation des substances humiques par hydrolyse basique et oxydation par le permanganate de potassium (figure 1).

Le caractère aromatique des matières humiques est reconnu par de nombreux auteurs comme responsable de la forte réactivité de ces composés avec les oxydants tels que le chlore et lozone.

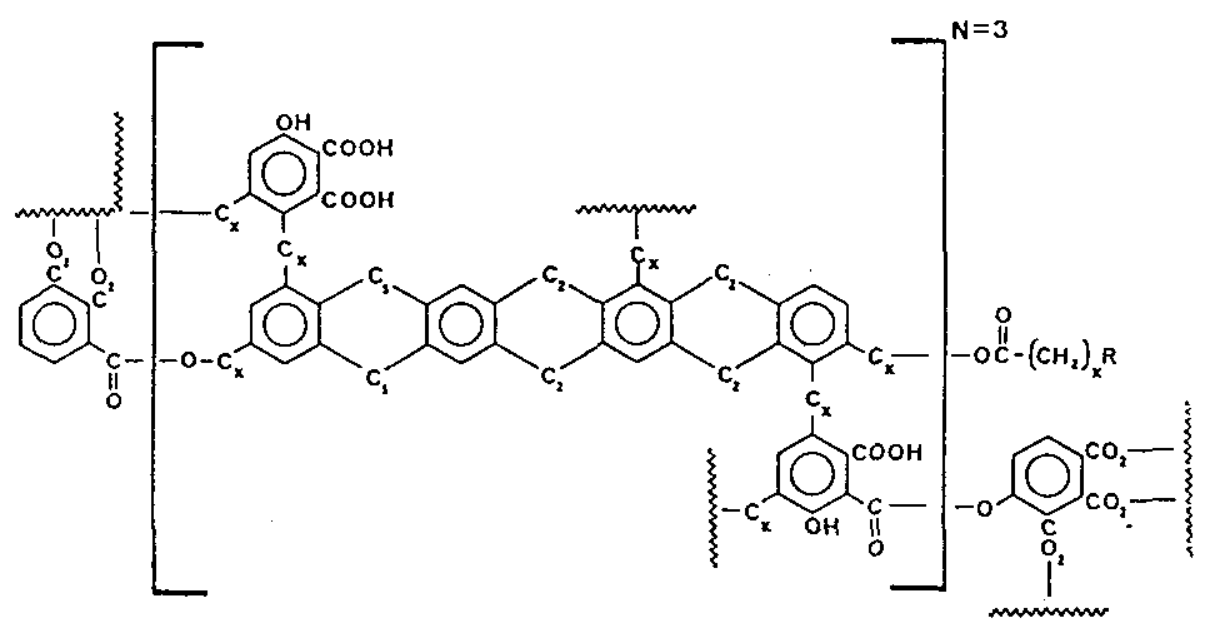

Figure 1 Modèle de structure de la matrice aromatique d'acides humiques d'origine aquatique d'aprés CHRISTMAN et al. (1987).

"Aromatic Matrix " model for aquatic humic macromolecular structure proposed by CHAISTMAN et al. (1987). 
La réactivité des substances humiques avec le chlore se traduit par une forte demande en oxydant, une diminution de la couleur et la formation de nombreux produits chlorés, exprimés globalement sous le terme de composés organohalogénés totaux (CIOT $\approx$ TOX) et des produits non chlorés. Le chloroforme et les acides dichloro et trichloroacétiques sont les principaux sous-produits de chloration analysables formés par chloration de substances humiques aquatiques (JOHNSON et al., 1982 ; MILLER et UDEN, 1983 ; NOR WOOD et al., 1983 ; COLEMAN et al., 1984 ; RECKHOW, 1984 ; SEEGER et al., 1984 ; CROUE, 1987). Certains des composés organo-chlorés formés présentent des propriétés mutagènes (DE GREEF et al., 1980 ; KRINGSTAD et al., 1983 ; COLEMAN et al., 1984 ; KOOL ef al., 1984 ; MEIER et al., 1985) et en particulier le chloro-3 (dichloromethyl) -4 hydroxy $-5(5 \mathrm{H})$ furanone -2 ou « $M X$ » et son isomère * $E-M X$ *, récemment identifiés lors de la chloration des eaux et des solutions de substances humiques (HEMMING et al., 1986 ; KRONBERG et al., 1987). L'essentiel des travaux de recherche sur les substances humiques aquatiques a été effectué par des laboratoires américains. Dans le but de contribuer à l'étude de leur structure et de leur comportement dans les étapes de traitement des eaux potables, nous avons entrepris depuis quelques années l'extraction de substances humiques d'eaux superficielles de plusieurs régions françaises.

De nombreux thèmes de recherche sur les substances humiques se sont développés dans notre laboratoire : action des oxydants comme le chlore et l'ozone (LEGUBE et al., 1985 et 1987 ; RECKHOW et al., 1986 ; CROUE, 1987), le bioxyde de chlore (BEN AMOR, 1988), les oxydants combinés UV-peroxyde d'hydrogène (GUITTONNEAU, 1986), ozone-peroxyde d'hydrogène (PAILLARD et al., 1988), l'adsorption sur charbon actif (LEGUBE et al., 1987 ; DE LAAT, 1988) et la coagulation-floculation (LEGUBE ot al., 1988 ; LEFEBVRE et al., 1989).

Nous présentons dans ce texte les résultats obtenus sur:

- les rendements d'extraction (et les techniques) des substances humiques issues de 11 eaux de surface différentes, dont 9 alimentent des usines d'eau potable, extraits,

- l'évaluation de quelques paramètres structuraux des acides fulviques

- la réactivité de ces acides fulviques avec le chlore.

\section{MATÉRIEL ET MÉTHODES}

\section{Méthode d'extraction utilisée}

Nous avons choisi d'extraire les substances humiques à partir de la méthode établie par THURMAN et MALCOLM (1981), avec quelques modifications mineures. Cette méthode comprend globalement deux étapes (fig. 2). 


\section{EAUNATURELIE \\ (stockage $4^{\circ} \mathrm{C}$ )}

FILTRATIONS SUCCESSIVES SUR MEMBRANES TUBULAIRES

$(10 \mu \mathrm{m} \mathrm{ct} 0,5 \mu \mathrm{m})$

$$
\downarrow
$$

ACIDIFICATION PAR ACIDE CHLORHYDRIQUE ( $\mathrm{pH}=2$ )

$\downarrow$

DEGAZAGE DU $\mathrm{CO}_{2}$

FILTRATION SUR XAD 8

$\downarrow$

REACIDIFICATION $\mathrm{pH}=2$

(*)

ELUTION PAR SOUDE $0,1 \mathrm{~N}$

ACIDIFICATION DE L'ELUAT SODIQUE (500 $\mathrm{mg} \mathrm{l}^{-1}$ COD)

PAR ACIDE CHLORHYDRIQUE $(\mathrm{pH}=1)$

PRECIPITATION (24 heures)

$\downarrow$

CENTRIFUGATION

RECUPERATION DU PRECIPITE

(ACIDES HUMIQUES)

RINCAGE EAU ULTRA PURE

$\downarrow$

REDISSOLUTION DANS SOUDE 0,1 N

FILTRATION SUR

ECHANGEUR DE CATIONS

$\downarrow$

LYOPHILISATION

$\downarrow$

ACIDES HUMIQUES

(forme acidc)

\section{RECUPERATIONDU SURNAGEANT (ACIDES FULVIQUES)}

FLTRATION SUR XAD 8 $\downarrow$

LAVAGE AVEC EAU ULTRA PURE $\downarrow$

ELUTION AVEC SOUDE 0,1 N

1

FILTRATION SUR

ECHANGEUR DE CATIONS

LYOPHILISATION

ACIDES FULVIQUES

(forme acidc)

contrôles analytiques: $\mathrm{COT} ; \mathrm{Na}^{+} ; \mathrm{UV}$ à $254 \mathrm{~nm}$

$\left(^{*}\right)$ : plusicurs ćtapes de concentration sur XAD 8 peuvent ĉtre effectuées jusqu'à l'obtention d'un éluat sodique de concentration $=500 \mathrm{mg} \mathrm{l}^{-1} \mathrm{COD}$.

Figure 2 Modèle d'extraction des substances humiques d'une eau naturelle selon la méthode de THURMAN et MALCOLM (1981) avec quelques modifications mineures.

Preparative isolation of humic substances from natural waters proposed by THUPMAN and MALCOLM (1981) with minor modifications.

Les conditions opératoires retenues pour l'étape d'adsorption-élution sont les suivantes :

- volume de résine XAD 8: 7 litres de résine préconditionnée selon la procédure décrite figure 3 ;

- débit de filtration sur XAD $8: 101 \mathrm{~h}^{-1}$ eau préfiltrée et à $\mathrm{pH}=2$;

- débit d'élution : $3 / h^{-1} \mathrm{NaOH} \mathrm{N/10}$. 


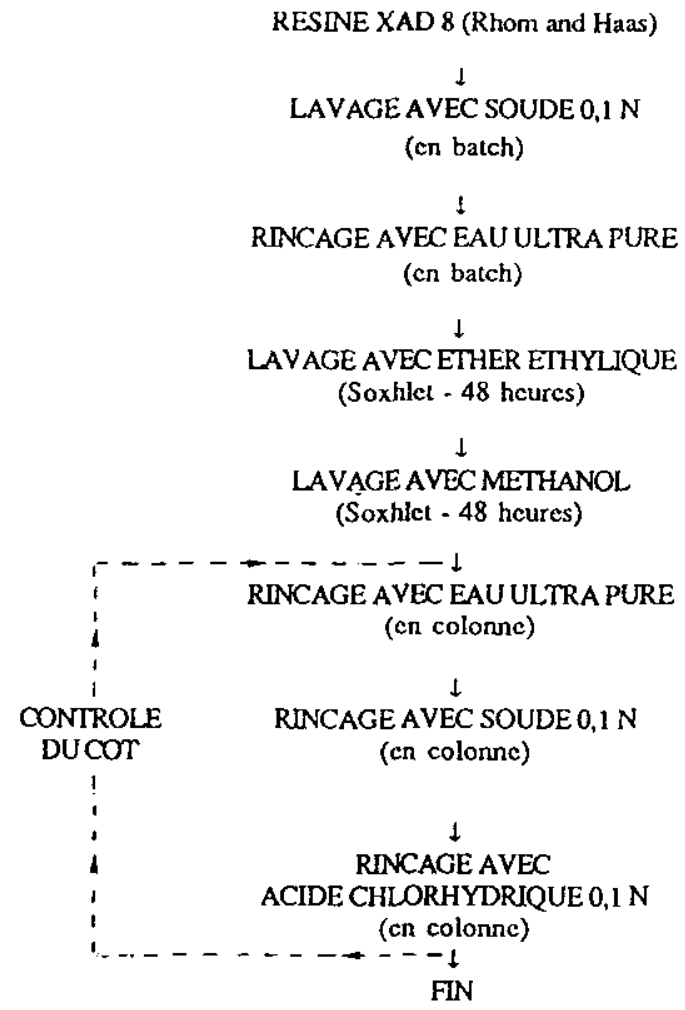

Figure 3 Conditionnement de la résine XAD 8 selon la méthode de THURMAN et MALCOUM (1981).

XAD 8 resin cleanup procedures (THURMAN and MALCOLM, 1981).

\section{Techniques analytiques}

\section{Analyse élémentaire}

Les analyses élémentaires ont été réalisées par le laboratoire d'analyse du CNRS de Solaize (Lyon) et ne prennent en compte que le carbone, I'hydrogène, l'oxygène, l'azote, le soufre et parfois le résidu selon la quantité d'échantillon disponible.

\section{Spectrométrie UV et IR}

Les spectres d'absorption UV des acides fulviques dissous dans un tampon phosphate (entre 35 et $80 \mathrm{mg} \mathrm{I}^{-1}$ AF ; $\mathrm{pH}=7,5$; force ionique $=10^{-2} \mathrm{M}$ ) ont été effectués avec un spectrophotomètre Varian DMS 90, dans des cuves en quartz de $1 \mathrm{~cm}$ de largeur. Les différentes mesures de densité optique ont été réalisées sur le même appareil (trajet optique de 1 ou $5 \mathrm{~cm}$ ). 
La spectrométrie infrarouge (en phase solide) a été conduite sur un spectromètre de type NIC $10 \mathrm{MX}$, pour des teneurs massiques en acide fulvique comprises entre 3 et $5 \%$ dans le $\mathrm{KBr}$.

\section{Fractionnement par ultrafiltration}

Des solutions d'acides fulviques de concentration $10 \mathrm{mg} \mathrm{t}^{-1} \mathrm{C}$ dans l'eau Milli Q (250 ml) ont été ultrafiltrées à l'aide d'une cellule “ Amicon " modèle 402 (capacité $400 \mathrm{ml}$ ) sous pression de 3 bars d'azote, par passage des membranes « Amicon " de type YC 05 ; YM 2 ; YM 10 et YM 30. Ces membranes possèdent des seuils de coupure apparents, respectivement égaux à $0.5 ; 1 ; 10$ et 30 kiloDaltons.

Avant l'utilisation, les membranes sont abondamment rincées à l'eau Milli $Q$ afin d'éliminer leur couche de glycérine protectrice. Une fois la membrane placée dans la cellule d'ultrafiltration, celle-ci est préalablement lavée avec $200 \mathrm{ml}$ d'eau Milli Q. Suite à cette opération, les 25 premiers millilitres d'échantillon passés à travers la membrane ne sont pas recueillis pour éviter toute dilution de l'ultrafiltrat. Comme certains travaux (OGURA, 1974 ; AIKEN, 1984) ont montré que l'augmentation progressive de la concentration en carbone organique dans le rétentat entraine le passage des composés de masse théoriquement inférieure au seuil de coupure de la membrane, nous n'avons récupéré qu'une seule partie des ultrafiltrats, soit environ $175 \mathrm{ml}$.

\section{Analyse des fonctions carboxyles}

Les fonctions carboxyles présentes dans la structure des acides fulviques ont été déterminées par un titrage potentiométrique par la soude $0,02 \mathrm{~N}$ sur $20 \mathrm{ml}$ de solution concentrée en acide fulvique ([AF] $=450 \mathrm{mg} \mathrm{l-1,} \mathrm{KCl}$ : $0,1 \mathrm{M}$ ). Par suivi du pH (pHmètre Tacussel Minisis 8000 ), sous courant d'azote, la concentration en fonctions carboxyles est obtenue pour un $\mathrm{pH}$ de titration égal à 8 . A ce $\mathrm{pH}$, une partie des composés phénoliques orthosubstitués peut être également prise en compte; celle-ci ne représente selon THURMAN (1985) qu'environ $10 \%$ de l'ensemble des fonctions dosées à ce pH.

\section{Analyse des potentiels de réactivité avec le chlore}

Pour les potentiels de formation (notés PF) des THM, TCA, DCA, TOX et les potentiels de demande en chlore, nous avons suivi le protocole suivant :

- concentration en acide fulvique $=5 \mathrm{mg}^{-1} \mathrm{AF}$,

- taux de chloration $=10 \mathrm{mg} \mathrm{I-} \mathrm{Cl}_{2}$,

$-\mathrm{pH}$ de chloration $=7,5$,

- force ionique $=10^{-2} \mathrm{M}$ (phosphates),

- temps de réaction $=72$ heures,

- obscurité à $20^{\circ} \mathrm{C}$.

Après 72 heures, le chlore résiduel est dosé par iodométrie (RODIER, 1984 ; CROUE, 1987) puis réduit par du méta-arsenite de sodium. Pour le dosage des trihalométhanes, $60 \mathrm{ml}$ de mélange réactionnel sont introduits 
dans un flacon pénicilline, rempli sans bulles d'air, bouché et capsulé. Une extraction au pentane est alors réalisée sous agitation pendant 30 minutes. Les différents THM sont séparés en chromatographie phase gazeuse (Packard 438.S) sur une colonne $10 \%$ FFAP/Chromosorb WAW 80 100 Mesh (DI $=2 \mathrm{~mm}, L=1,8 \mathrm{~m}$ ) et dosés par un détecteur à capture d'élections (source ${ }^{63 N i)}$ (CROUE, 1987).

La méthode d'analyse des acides trichloroacétique (TCA) et dichloroacétique (DCA) consiste en une extraction par l'éther éthylique en deux étapes (à pH neutre puis acide) suivie d'une concentration, d'une méthylation par le diazométhane et d'une analyse chromatographique réalisée dans les mêmes conditions que pour les THM. De nombreux détails sur cette méthode d'analyse ont été publiés ailleurs (RECKHOW, 1984 ; CROUE, 1987).

Les erreurs relatives sur l'analyse des potentiels de réactivité avec le chlore ont été estimées à $\pm 10 \%$ pour les THM et l'acide DCA et de $\pm 5 \%$ pour l'acide TCA.

Les TOX sont analysés sur un appareil Dohrman. 1987)

Analyse par couplage CG/SM des produits de réaction avec le chlore (CRouE,

Les produits de chloration ont été extraits par l'éther éthylique à partir d'une solution d'acide fulvique « Pinail * à $1 \mathrm{~g} \mathrm{~F}^{-1}(\mathrm{pH}=7,5$; force ionique $=10^{-1} \mathrm{M}$; phosphates) chlorée (ou non chlorée pour le blanc) à $3,6 \mathrm{mg}$ de chlore par $\mathrm{mg}$ de carbone pendant 72 heures, puis acidifiée à $\mathrm{pH}=1$ par l'acide chlorhydrique. Après concentration à l'évaporateur rotatif, les extraits de la solution chlorée et du blanc sont séchés sur sulfate de sodium anhydre et méthylés au diazométhane. Le volume final des extraits est réduit à $1 \mathrm{ml}$ sous courant d'azote purifié (four Supelco). Les conditions de chromatographie gazeuse et de spectrométrie de masse sont mentionnées sur le chromatogramme reconstitué, présenté figure 9. L'analyse finale a été réalisée par le Centre de Spectrométrie de Masse du Laboratoire d'Analyse du CNRS de Solaize.

\section{Divers}

- L'eau désignée eau Milli $Q$ utilisée dans toutes les techniques analytiques, ainsi que pour les solutions de substances humiques, est produite par une unité Millipore (Milli RO puis Milli Q), avec une résistivité de $18106 \Omega \mathrm{cm}$ et une teneur en carbone organique intérieure ou égale à $100 \mu \mathrm{g} \mathrm{I}^{-1}$.

- Tous les sels utilisés sont des réactifs purs de qualité analytique et, dans le cas de l'analyse par couplage CG/SM, sont préalablement purifiés par extraction au soxhlet à l'éther éthylique (24 heures) puis séchés à l'étuve.

- Le diazométhane est synthétisé au laboratoire à partir du $\mathrm{N}$-nitroso-N-méthyle-p. toluène sulfamide (VOGEL, 1956).

- Les analyses de COT sont effectuées sur un appareil Dorhman DC 80 , par oxydation UV-persulfate. 


\section{RÉSULTATS ET DISCUSSION}

\section{Extraction des substances humiques}

Les eaux de surface à partir desquelles ont été extraites les substances humiques, ont été choisies en fonction de leur teneur élevée en matière organique d'origine naturelle (cas des eaux fortement colorées) et de leur utilisation pour la production d'eau potable. Huit des eaux sélectionnées répondent à ces deux critères (tableau 1). Les eaux « Les Landes » et “Pinail " sont des eaux d'étang et de mare situés dans des sites protégés. L'eau de la Seine est utilisée pour la production d'eau potable mais contient peu de matière organique par comparaison avec les autres eaux.

Le tableat 1 présente les origines et les teneurs en matière organique des eaux étudiées ainsi que les volumes et les rendements d'extraction obtenus.

Les rendements d'extraction sont faibles et compris entre 3 et $12 \mathrm{~g} \mathrm{SH} / \mathrm{m}^{3}$ eau brute (16 à $30 \% \mathrm{C} / \mathrm{COD}$ ) à l'exception de l'eau de la Seine pour laquelle le rendement est seulement de $0,06 \mathrm{~g} \mathrm{SH} / \mathrm{m3}$ (1,3\% C/COD). En outre, le rendement d'extraction des substances humiques d'une eau dépend des conditions opératoires utilisées et en particulier du rapport volume d'eau traitée/volume de résine. En effet, dans le cas des extractions des eaux *beaufort *, « Gatineaux * et * Cebron *, nous avons montré figure 4 que les rendements d'extraction par la résine XAD 8 décroissent rapidernent avec l'augmentation du volume traité. Par conséquent, les concentrations en substances humiques déduites de ces travaux (rendement d'extraction en $\mathrm{g} / \mathrm{m}^{3} \mathrm{~EB}$ ) représentent des valeurs par défaut.

L'origine des eaux est également un facteur prépondérant puisque le rendement d'extraction est directement proportionnel à l'absorbance UV de l'eau brute apres filtration à $0,5 \mu \mathrm{m}$, pour le cas d'extractions comparables, c'est-à-dire pour des rapports voisins de volume d'eau traitée sur volume de résine (fig. 5).

L'influence de ces deux paramètres peuvent expliquer en partie le faible rendement observé pour l'eau de Seine.

Notons que la fraction acide fulvique représente, en pourcentage massique 60 à $80 \%$ (tableau 1) des substances humiques (excepté Seine), démontrant ainsi la prédominance de cette fraction comme lindique THURMAN (1985). Les matières humiques de l'eau de Seine seraient essentiellement constituées d'acides fulviques, résultat discutable qui pose le problème de la nature exacte des produits extraits par cette méthode dans le cas d'une telle eau.

De ces résultats découlent deux inconvénients, le premier d'ordre purement matériel est qu'il est nécessaire de manipuler de grands volumes d'eau pour obtenir quelques grammes de substance humique dans des temps importants (4 semaines pour l'extraction et la purification à partir d'un $\mathrm{m}^{3}$ d'eau brute). Le second inconvénient est d'ordre technique; en effet, on peut s'interroger sur le degré d'altération des substances humiques engendré par le processus d'extraction (AIKEN, 1988). En d'autres termes, on peut se demander si les résultats (de chloration, d'ozonation, de coagulationfloculation, etc.) obtenus avec des solutions synthétiques de substance humique sont totalement transposables aux cas des eaux brutes. 
Tableau 1 Origines et rendements d'extraction des substances humiques étudiées.

Table 1 Extraction yields and localisation of the studied humic substances.

\begin{tabular}{|c|c|c|c|c|c|c|c|c|}
\hline $\begin{array}{l}\text { Lleu de prelèvement } \\
\text { Pérlode de } \\
\text { prelévement }\end{array}$ & Ortgine & Abreviatlon & $\begin{array}{l}\text { Vol. d'eau traltée } \\
\mathrm{m}^{3}\end{array}$ & $\begin{array}{l}\operatorname{COD}(* *) \\
m g H^{-1} C\end{array}$ & $\begin{array}{l}\text { UV }\left({ }^{* *}\right) \\
254 \mathrm{~nm}, 1 \mathrm{~cm}\end{array}$ & $\begin{array}{l}\text { Rendement } \\
\text { d'extraction } \\
\mathrm{o} \mathrm{SH} / \mathrm{m}^{3} \mathrm{~EB}\end{array}$ & $\begin{array}{l}\text { Rapport } \\
\text { massique } \\
\text { AF/SH \% }\end{array}$ & $\begin{array}{l}\text { Rendement } \\
\text { d'extraction } \\
\text { C/COO } \%\end{array}$ \\
\hline $\begin{array}{l}\text { Beaufort, barrage } \\
\text { Février } 88\end{array}$ & (") St Malo (35) CEO & B & 0,85 & 8,2 & 0,292 & 5,9 & 78,7 & 34,5 \\
\hline $\begin{array}{l}\text { Cebron, barrage } \\
\text { Novembre } 87\end{array}$ & (") Cebron (79), CGE & C & 1,0 & 15,1 & 0,408 & 7,3 & 81,1 & 24,1 \\
\hline $\begin{array}{l}\text { Gartempe, ivilère } \\
\text { Juin } 87\end{array}$ & (") Beilac (87), CGE & G & 0,925 & 9,9 & 0,312 & 4,2 & 70,6 & 21,7 \\
\hline $\begin{array}{l}\text { Gatineaux, barrage } \\
\text { Mars } 88\end{array}$ & (") Pornic (44), CEO & Gat & 0,875 & 14,4 & 0,556 & 12,3 & 64,8 & 35,2 \\
\hline Les Landes & $\begin{array}{l}\text { Près de Bordeaux (33) } \\
\text { Lac Gazinet }\end{array}$ & $L$ & 0,25 & 8 & & 2,9 & 87,4 & 18,8 \\
\hline $\begin{array}{l}\text { Moulin Papon, banrage } \\
\text { Octobre } 87\end{array}$ & (") La Roche-sur-Yon (85), & MP & 0,80 & 9 & & 4,4 & 66 & 22,8 \\
\hline Pinail, mare & Forêt près de Poitiers (86) & $P$ & 2,5 & 15 & & 6,2 & 74,2 & 22,4 \\
\hline Seine, fleuve & $\begin{array}{l}\text { Maisons Laffitte (78) } \\
\text { Anjou-Recherche }\end{array}$ & Se & 90 & 2,4 & 0,037 & 0,06 & 97,9 & 1,3 \\
\hline $\begin{array}{l}\text { Sorme, barrage } \\
\text { Novembre } 87\end{array}$ & $\begin{array}{l}\text { (")Montceatr } \\
\text { les-mines (71), CGE }\end{array}$ & So & 1,0 & 8,6 & 0,180 & 2,7 & 81,7 & 16,0 \\
\hline $\begin{array}{l}\text { Vienne, iviare } \\
\text { Avril } 87\end{array}$ & $\begin{array}{l}\text { (*) Chattellerault (86) } \\
\text { CEO }\end{array}$ & v & 0,75 & 6,9 & 0,170 & 3,0 & 63,8 & 21,2 \\
\hline $\begin{array}{l}\text { Villejean, barrage } \\
\text { Mars } 87\end{array}$ & (") Rennes (35), CGE & Vi & 1,0 & 7,2 & 0,211 & 3,2 & 79,9 & 22,5 \\
\hline
\end{tabular}

EB : eau brute ; SH ; substancess humiques; AF : acide fulvique ; $C O D$ : carbone organique dissous ; $C$; carbone calculéa à partir de l'analyse élémentaire (tableau 2 ) et en tenant compte des proportions

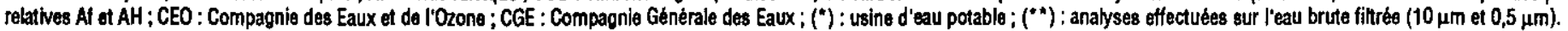



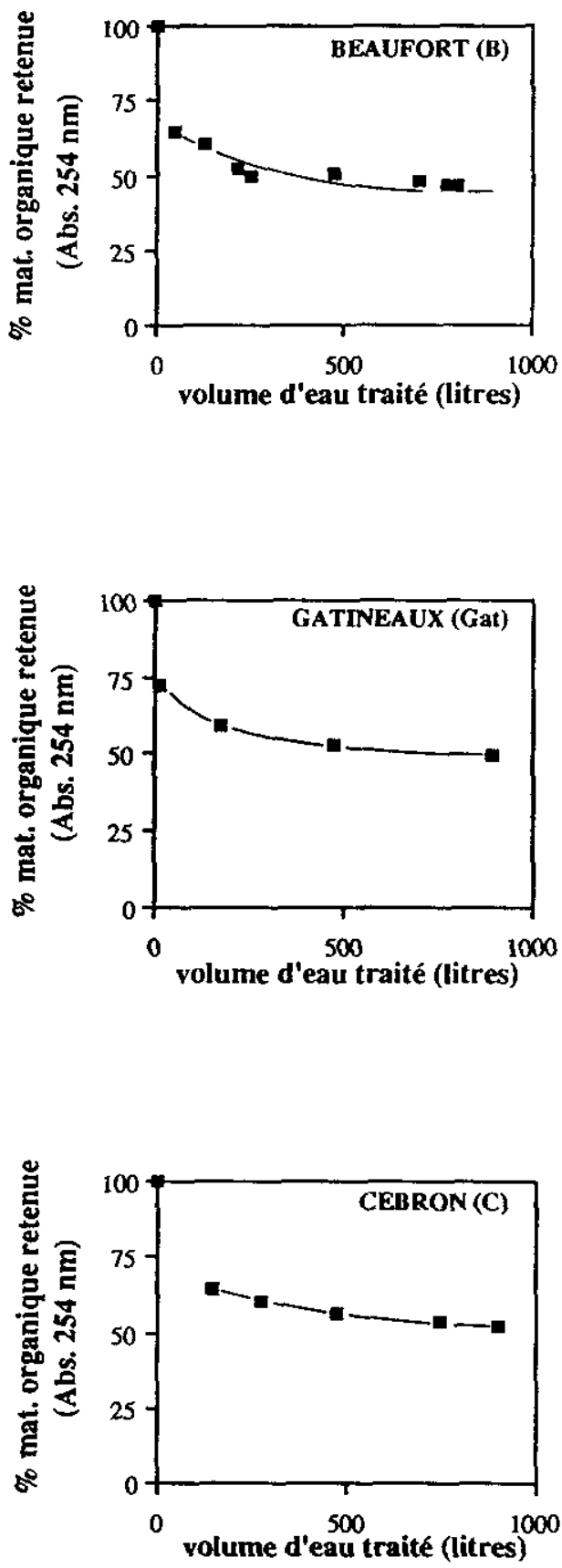

Figure 4 Pourcentage de matière organique retenue par la résine XAD 8 en fonction du volume ơeau passé.

Dissolved organic matter recovery for $X A D \&$ resin as a function of the used water volume. 


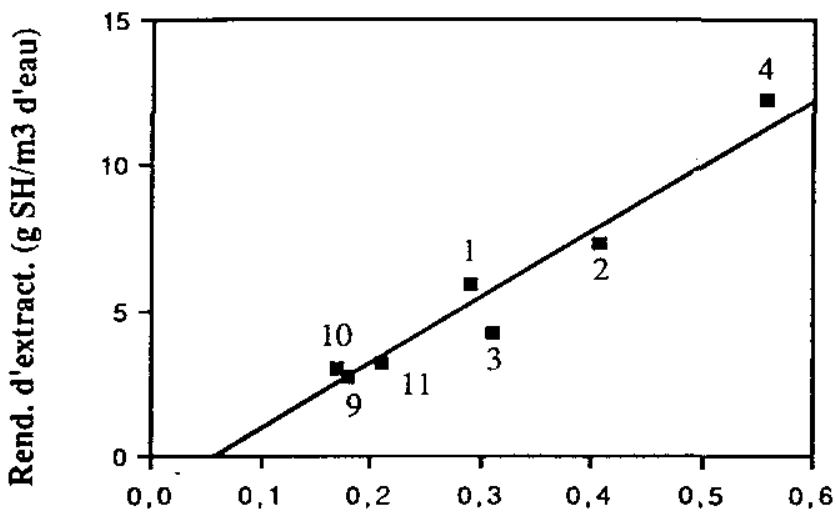

Abs. (254 nm) des eaux brutes filtrées à 0,5 um (cm-1)

Figure 5 Corrélation entre le rendement d'extraction des substances humiques et l'absorption UV (254 $\mathrm{nm})$ des eaux brutes filtrées

(1: AFB ; $2:$ AFC ; $3:$ AFG ; $4:$ AFGat ; $9:$ AFSo ; $10:$ AFV ; 11 : AFVj).

Correlation between humic substance extraction yields and UV absorbance $(254 \mathrm{~nm})$ of the filtered natural waters

(1: AFB ; 2 : AFC ; $3:$ AFG ; $4:$ AFGat ; $9:$ AFSo ; $10:$ AFV ; 11 : AFV $)$.

\section{Caractérisation des substances humiques}

Dans le tableau 2 sont regroupées les différentes analyses élémentaires.

Pour tous les acides fulviques extraits, les pourcentages en carbone, hydrogène et oxygène sont à peu près identiques. Par contre, les teneurs en soufre des acides fulviques Vienne et Seine sont beaucoup plus élevées que dans les autres cas.

Les teneurs en résidus des acides humiques sont fortes et globalement de l'ordre de $10 \%$, valeurs nettement supérieures à celles obtenues pour les acides fulviques. En conséquence, la comparaison de ces différentes analyses élémentaires ne peut $s e$ justifier qu'après déduction du pourcentage de résidu. Dans ce cas on constate essentiellement que les acides humiques présentent une teneur en azote plus élevée que les acides fulviques, observation en accord avec les données bibliographiques (THURMAN, 1985).

Par comparaison, les substances humiques commerciales contiennent une quantité importante de résidu et beaucoup moins d'azote que les substances humiques naturelles aquatiques. En conséquence, leur utilisation pour évaluer la nature et la réactivité des substances humiques dans les eaux naturelles n'est peut-être pas toujours bien appropriée, comme l'ont signalé MALCOLM ET MAC CARTY (1986). 
Tableau 2 Analyses élémentaires des acides fulviques et humiques étudiés. Comparaison avec les substances humiques commerciales.

Table 2 Elementary analysis of the studied humic and fulvic acids. Comparison with commercial humic substances.

\begin{tabular}{|c|c|c|c|c|c|c|}
\hline Substances humlques & $\% \mathrm{C}$ & $\% \mathbf{H}$ & $\% N$ & $\% 0$ & $\% S$ & \% Résldu \\
\hline $\begin{array}{l}\text { AFB } \\
\text { AHB }\end{array}$ & $\begin{array}{l}49,07 \\
43,85\end{array}$ & $\begin{array}{l}4,98 \\
5,28\end{array}$ & $\begin{array}{l}1,68 \\
2,86\end{array}$ & $\begin{array}{l}39,66 \\
31,22\end{array}$ & $\begin{array}{l}1,18 \\
0,74\end{array}$ & $\begin{array}{r}3,43\left(^{*}\right) \\
16,05\left(^{*}\right)\end{array}$ \\
\hline $\begin{array}{l}\text { AFC } \\
\text { AHC }\end{array}$ & $\begin{array}{l}50,75 \\
46,30\end{array}$ & $\begin{array}{l}5,05 \\
4,92\end{array}$ & $\begin{array}{l}1,77 \\
2,88\end{array}$ & $\begin{array}{l}38,90 \\
34,96\end{array}$ & $\begin{array}{l}1,31 \\
1,04\end{array}$ & $\begin{array}{l}2,22 \quad\left(^{*}\right) \\
9,9 \quad(*)\end{array}$ \\
\hline $\begin{array}{l}\text { AFG } \\
\text { AHG }\end{array}$ & $\begin{array}{l}52,20 \\
47,22\end{array}$ & $\begin{array}{l}5,08 \\
5,12\end{array}$ & $\begin{array}{l}1,71 \\
2,56\end{array}$ & $\begin{array}{l}37,75 \\
36,14\end{array}$ & $\begin{array}{l}0,59 \\
1,00\end{array}$ & $\begin{array}{l}2,67\left(^{*}\right) \\
5,00(*)\end{array}$ \\
\hline $\begin{array}{l}\text { AFGat } \\
\text { AHGat }\end{array}$ & $\begin{array}{l}49,06 \\
26,92\end{array}$ & $\begin{array}{l}4,88 \\
3,51\end{array}$ & $\begin{array}{l}1,87 \\
1,57\end{array}$ & $\begin{array}{l}37,46 \\
28,93\end{array}$ & $\begin{array}{l}1,57 \\
0,61\end{array}$ & $\begin{array}{r}5,16 \quad(*) \\
38,46 \quad(*)\end{array}$ \\
\hline $\begin{array}{l}\text { AFL } \\
\text { AHL }\end{array}$ & $\begin{array}{l}53,4 \\
46,6\end{array}$ & $\begin{array}{l}5,3 \\
4,0\end{array}$ & $\begin{array}{l}1,1 \\
1,75\end{array}$ & $\begin{array}{l}30,0 \\
-\end{array}$ & - & $\overline{-}_{10,0}$ \\
\hline $\begin{array}{l}\text { AFMP } \\
\text { AHMP }\end{array}$ & $\begin{array}{l}51,79 \\
37,8\end{array}$ & $\begin{array}{l}5,28 \\
5,5\end{array}$ & $\begin{array}{l}1,76 \\
3,2\end{array}$ & $\begin{array}{l}37,9 \\
38,1\end{array}$ & $\begin{array}{l}1,28 \\
-\end{array}$ & $\begin{array}{c}1,99\left({ }^{*}\right) \\
12,0 \quad(*)\end{array}$ \\
\hline $\begin{array}{l}\text { AFP } \\
\text { AHP }\end{array}$ & $\begin{array}{l}54,6 \\
52,65\end{array}$ & $\begin{array}{l}4,8 \\
5,3\end{array}$ & $\begin{array}{l}0,8 \\
1,8\end{array}$ & $\begin{array}{l}37,7 \\
33,1\end{array}$ & - & ${ }^{-} 6,3 \quad(* *)$ \\
\hline $\begin{array}{l}\text { AFSe } \\
\text { AHSe }\end{array}$ & $\begin{array}{l}51,70 \\
50,63\end{array}$ & $\begin{array}{l}5,65 \\
5,50\end{array}$ & $\begin{array}{l}1,50 \\
4,42\end{array}$ & $\begin{array}{l}38,39 \\
32,88\end{array}$ & $\begin{array}{l}2,40 \\
1,92\end{array}$ & $\begin{array}{ll}2,0 & (* *) \\
1,2 & (* *)\end{array}$ \\
\hline $\begin{array}{l}\text { AFSo } \\
\text { AHSo }\end{array}$ & $\begin{array}{l}51,83 \\
43,76\end{array}$ & $\begin{array}{l}5,65 \\
5,49\end{array}$ & $\begin{array}{l}1,71 \\
3,73\end{array}$ & $\begin{array}{l}37,94 \\
33,63\end{array}$ & $\begin{array}{l}1,06 \\
1,50\end{array}$ & $\begin{array}{r}\left.1,81 \stackrel{*}{*}^{*}\right) \\
11,89(*)\end{array}$ \\
\hline $\begin{array}{l}\text { AFV } \\
\text { AHV }\end{array}$ & $\begin{array}{l}50,21 \\
45,2\end{array}$ & $\begin{array}{l}4,86 \\
4,92\end{array}$ & $\begin{array}{l}1,00 \\
2,46\end{array}$ & $\begin{array}{l}38,48 \\
33,83\end{array}$ & $\begin{array}{l}5,13 \\
1,75\end{array}$ & $<1 \quad(* *)$ \\
\hline $\begin{array}{l}A F V j \\
A H V j\end{array}$ & $\begin{array}{l}52,26 \\
45,6\end{array}$ & $\begin{array}{l}5,21 \\
5,60\end{array}$ & $\begin{array}{l}1,33 \\
5,07\end{array}$ & $\begin{array}{l}38,79 \\
33,65\end{array}$ & $\begin{array}{l}0,71 \\
1,22\end{array}$ & $\begin{array}{l}1,70 \quad \text { (") } \\
8,0 \quad(*)\end{array}$ \\
\hline SH FLUKA & 38,23 & 4,45 & 0,43 & 34,45 & 2,39 & 26 \\
\hline SH ALDRICH & 36,94 & 4,24 & 0,46 & 38,76 & 2,13 & $(*)$ \\
\hline
\end{tabular}

(i) Résidu calculê : complément à 100.

(i) Résidu analysé.

Pour des longueurs d'onde comprises entre 200 et $400 \mathrm{~nm}$, les acides fuiviques ne présentent pas de bande d'absorption spécifique et les spectres traduisent une augmentation progressive de l'absorbance vers les faibles longueurs d'onde. Toutefois, l'absorbance UV rapportée à l'unité de masse d'acide fulvique (ou de carbone) varie dans des proportions significatives selon l'origine des eaux étudiées (tableau 3).

Les spectres en infrarouge sont identiques entre eux (pour trois acides fulviques étudiés : acides fulviques “Landes „ ou AFL, « Moulin Papon ^. ou AFMP et * Pinail * ou AFP) et à ceux présentés dans la bibliographie (THURMAN, 1985). Ces spectres présentent trois zones de fréquences d'absorption caractéristiques, entre 3400 et $3200 \mathrm{~cm}^{-1}$ (liaison $(\mathrm{O}-\mathrm{H}$ ), aux environs de $2900 \mathrm{~cm}^{-1}$ (liaison aliphatique $\mathrm{C}-\mathrm{H}$ ) et vers $1720 \mathrm{~cm}^{-1}$ (liaison $\mathrm{C}=\mathrm{O}$ ). 
Tableau 3 Quelques paramètres de caractérisation des acides fulviques étudiés et leurs potentiels de réactivité avec le chlore.

Table 3 Characterization of the studied fulvic acids and their chlorine reactivity potentials.

\begin{tabular}{|c|c|c|c|c|c|c|c|}
\hline Acide fulvique & $\begin{array}{l}\text { Absortance } 254 \mathrm{~nm}, 5 \mathrm{~cm} \\
\text { rapportes au mg } \mathrm{L}^{-1} \mathrm{AF} \\
\text { (n) }\end{array}$ & $\begin{array}{l}\text { Fonctlons carboxyles } \\
\text { meq/g AF } \\
\text { (n) }\end{array}$ & $\begin{array}{l}\text { PF THM } \\
\mu g \mathrm{CHCl}_{3} / \mathrm{mg} \text { AF } \\
\text { (n) }\end{array}$ & $\begin{array}{l}\mathrm{P} \text { demande en } \mathrm{Cl}_{2} \\
\mathrm{mg} \mathrm{Cl} / \mathrm{mg} \mathrm{AF} \\
\text { (n) }\end{array}$ & $\begin{array}{l}\text { PF TOX } \\
\mu g \mathrm{Cl} / \mathrm{mg} \text { AF } \\
\text { (n)S }\end{array}$ & $\begin{array}{l}\mathrm{TOX} / \mathrm{Cl}_{2} \text { cons. } \\
\% \mathrm{Cl} / \mathrm{Cl}\end{array}$ & $\begin{array}{l}\mathrm{CHCl}_{3} / \mathrm{TOX} \\
\% \mathrm{CV} / \mathrm{Cl}\end{array}$ \\
\hline AF $B$ & $\begin{array}{l}0,0906 \\
(2)\end{array}$ & $\begin{array}{l}6,35 \\
(1)\end{array}$ & & $\begin{array}{l}0,78 \\
(2)\end{array}$ & $\begin{array}{l}78,9 \\
(2)\end{array}$ & 10,2 & \\
\hline $\mathrm{AFC}$ & $\begin{array}{l}0,0912 \\
(6)\end{array}$ & $\begin{array}{l}5,8 \\
\text { (1) }\end{array}$ & $\begin{array}{l}23,8 \\
(3)\end{array}$ & $\begin{array}{l}0,75 \\
(4)\end{array}$ & $\begin{array}{l}77,5 \\
(4)\end{array}$ & 10,3 & 27,4 \\
\hline$A F G$ & $\begin{array}{l}0,1024 \\
(6)\end{array}$ & $\begin{array}{l}6,8 \\
\text { (1) }\end{array}$ & $\begin{array}{l}30,3 \\
(6)\end{array}$ & $\begin{array}{l}0,76 \\
\text { (6) }\end{array}$ & $\begin{array}{l}86,4 \\
(4)\end{array}$ & 11,4 & 31,2 \\
\hline AF Gat & $\begin{array}{l}0,0982 \\
(2)\end{array}$ & $\begin{array}{l}6,7 \\
\text { (1) }\end{array}$ & & $\begin{array}{l}0,80 \\
\text { (2) }\end{array}$ & $\begin{array}{l}77,3 \\
(2)\end{array}$ & 9,6 & \\
\hline$A F L$ & $\begin{array}{l}0,0676 \\
(6)\end{array}$ & $\begin{array}{l}6,0 \\
(4)\end{array}$ & $\begin{array}{l}14,9 \\
(3)\end{array}$ & $\begin{array}{l}0,48 \\
\text { (3) }\end{array}$ & $\begin{array}{l}63,0 \\
(1)\end{array}$ & 13,2 & 21,1 \\
\hline AFMP & $\begin{array}{l}0,0834 \\
(6)\end{array}$ & $\begin{array}{l}5,2 \\
\text { (1) }\end{array}$ & $\begin{array}{l}19,3 \\
(2)\end{array}$ & $\begin{array}{l}0,52 \\
(1)\end{array}$ & $\begin{array}{l}54,5 \\
\text { (1) }\end{array}$ & 10,5 & 31,6 \\
\hline AF $P$ & $\begin{array}{l}0,1053 \\
(6)\end{array}$ & $\begin{array}{l}5,3 \\
(4)\end{array}$ & $\begin{array}{l}35,8 \\
(5)\end{array}$ & $\begin{array}{l}0,93 \\
(5)\end{array}$ & $\begin{array}{l}106,0 \\
(3)\end{array}$ & 11,5 & 30,1 \\
\hline AF Se & $\begin{array}{l}0,0712 \\
(6)\end{array}$ & $\begin{array}{l}6,3 \\
(1)\end{array}$ & $\begin{array}{l}18,3 \\
(6)\end{array}$ & $\begin{array}{l}0,62 \\
(4)\end{array}$ & $\begin{array}{l}59,3 \\
(2)\end{array}$ & 9,52 & 27,5 \\
\hline AF So & $\begin{array}{l}0,0953 \\
(6)\end{array}$ & $\begin{array}{l}5,6 \\
\text { (1) }\end{array}$ & $\begin{array}{l}26,6 \\
\text { (3) }\end{array}$ & $\begin{array}{l}0,74 \\
(4)\end{array}$ & $\begin{array}{l}75,4 \\
(4)\end{array}$ & 10,2 & 31,4 \\
\hline AFV & $\begin{array}{l}0,1012 \\
(6)\end{array}$ & $\begin{array}{l}6,9 \\
(1)\end{array}$ & $\begin{array}{l}28,1 \\
(5)\end{array}$ & $\begin{array}{l}0,83 \\
(6)\end{array}$ & $\begin{array}{l}97,6 \\
(4)\end{array}$ & 11,8 & 25,7 \\
\hline AF V J & $\begin{array}{l}0,080 \\
(6)\end{array}$ & $\begin{array}{l}6,7 \\
(1)\end{array}$ & $\begin{array}{l}24,6 \\
(6)\end{array}$ & $\begin{array}{l}0,55 \\
(6)\end{array}$ & $\begin{array}{l}66,1 \\
(4)\end{array}$ & 12,0 & 33,2 \\
\hline
\end{tabular}

Af : acides fulviques ; $P$ : potentiel ; THM : trihalomethanes; TOX : orpanoctiorés totaux. (n) : nombre de points pris en compte pour le calcul de la moyenne. $P$ et $P F$ : potentiel de formation (AF $=5$ mglt 1 . $\mathrm{Cl}_{2}=10 \mathrm{mgh}-1 ; \mathrm{pH}^{2}=7,5 ; 72 \mathrm{hrs} ; 20^{\circ} \mathrm{C}$ ). 
La figure 6 présente la courbe de titrage par la soude des fonctions carboxyles de l'acide fulvique «Pinail" (AFP). La courbe dérivée permet de mieux visualiser le point de titration des fonctions carboxyles. Les résultats obtenus pour les 11 acides fulviques, mentionnés dans le tableau 3 , montrent une variation importante selon lorigine puisque la valeur moyenne est de l'ordre de $6 \mathrm{méq} / \mathrm{g}$ AF et les valeurs extrêmes 5,2 et $6,9 \mathrm{méq} / \mathrm{g} \mathrm{AF}$. Autrement dit, dans la structure d'un acide fulvique, 1 atome de carbone sur 7 est inclus dans une fonction carboxyle. On peut noter dans la bibliographie que les teneurs en fonctions carboxyles d'acides fuiviques extraits d'eaux américaines varient de 3,8 à 6,8 méq/g AF, résultats obtenus par titration par la soude et RMN ${ }^{13} \mathrm{C}$ du solide confondus (THURMAN et MALCOLM, 1983 ; OLIVER et THURMAN, 1983).

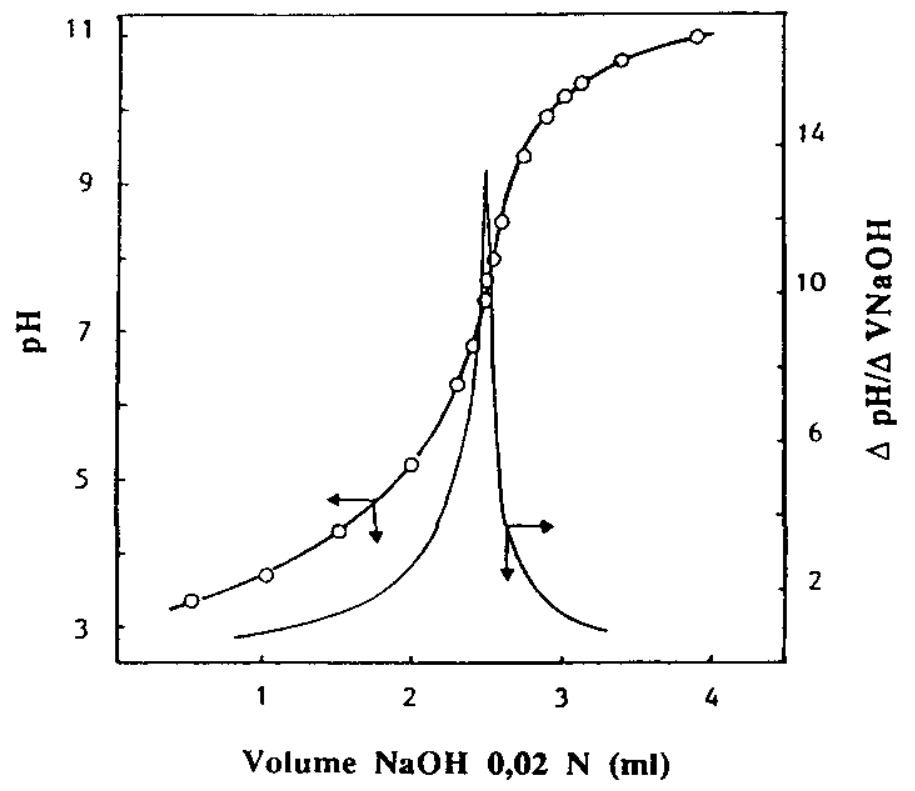

Figure 6 Courbe de titration par la soude des fonctions carboxyles de l'acide fulvique *Pinail " (AFP), à partir de $20 \mathrm{ml}$ d'une solution à $450 \mathrm{mg} \mathrm{AF} \mathrm{I-1} \mathrm{(dans} \mathrm{KCl} 0.1 \mathrm{M}$ ).

Carboxyl functions titration curve of the "Pinail " fulvic (AFP) $(20 \mathrm{ml}$ of a $450 \mathrm{mg} t^{1}$ AF solution in $\mathrm{KCl} 0,1 \mathrm{M}$.

Quelques expériences d'ultrafiltration ont été effectuées à partir de solutions dans l'eau Milli $Q$ de trois acides fulviques (AFL, AFMP et AFP). Les résultats exprimés en COD et en absorbance-UV $(270 \mathrm{~nm}, 1 \mathrm{~cm})$ dans l'ultrafiltrat, sont présentés sur la figure 7.

Le carbone organique des acides fulviques que nous avons extraits est constitué par 40 à $50 \%$ de composés de masse moléculaire apparente (MM ap.) < $10 \mathrm{kD}$. Les 50 à $60 \%$ restants sont représentés en majeure partie par des composés de MM ap. comprises entre 10 et $30 \mathrm{kD}$. Les mêmes remarques s'appliquent aux résultats obtenus par absorbance UV $270 \mathrm{~nm}$. 


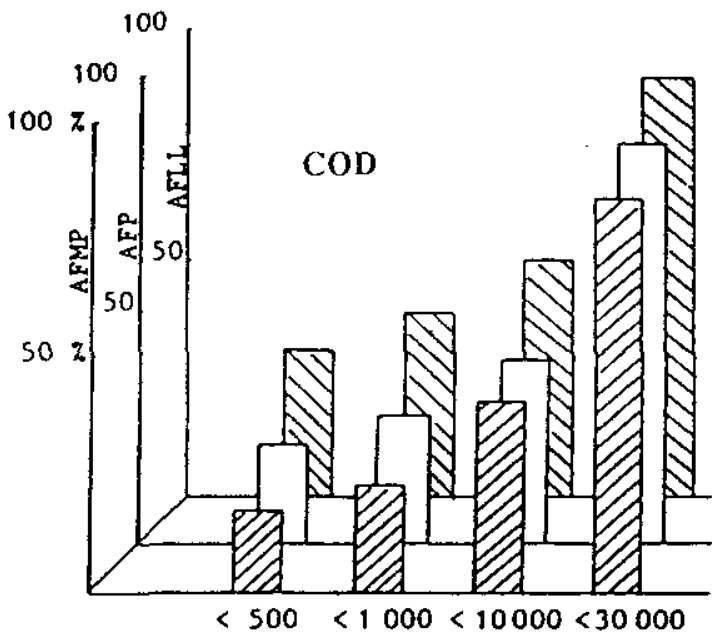

Masses moléculaires apparentes (daltons)

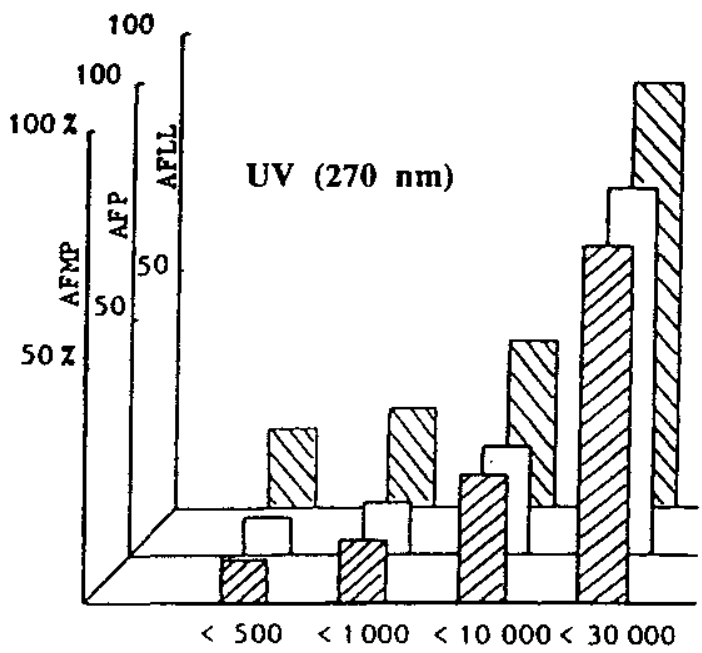

Masses moléculaires apparentes (daltons)

Figure 7 Ultrafiltration de solutions d'acides fulviques * Landes " (AFL), " Moulin Papon * (AFMP) et * Pinail * (AFP) $\left(10 \mathrm{mg} 1^{-1} \mathrm{C}\right.$, eau Milli Q) : degré d'aromaticité (absorbance UV à $270 \mathrm{~nm}$ ) et COT des différentes fractions étudiées.

Fractionation by ultrafiltration of the * Landes" (AFL), * Moulin Papon (AFMP) and * Pinail " (AFP) fulvic acids $\left(10 \mathrm{mg} r^{-1} \mathrm{C}\right.$, Milli $Q$ water) : UV absorbance $(270 \mathrm{~nm})$ and TOC of the studied fractions. 
Notons que ces résultats ne sont pas en accord avec la bibliographie qui mentionne des MM ap. généralement inférieures à $10 \mathrm{kD}$ pour les substances humiques aquatiques (THURMAN et MALCOLM, 1983 ; THURMAN, 1985). II est difficile de remettre en cause la méthode d'extraction des substances humiques que nous avons utilisée et il est peu probable que cette différence soit liée à la nature de nos acides fulviques, tous les autres résultats étant comparables à ceux de la bibliographie. L'écart de MM ap. observé est donc probablement dû à la technique d'ultrafiltration que nous avons utilisée, puisque de nombreux chercheurs ont montré que la détermination des MM ap. est fonction de divers paramètres comme la pression, le $\mathrm{pH}$, la force ionique et même les caractéristiques des membranes d'ultrafiltration (OGURA, 1974 ; AIKEN, 1984).

II est intéressant de noter que la valeur de l'absorbance UV à $270 \mathrm{~nm}$, rapportée à l'unité de concentration, croît significativement avec la masse moléculaire apparente des ultrafiltrats (tableau 4), montrant ainsi que les fractions des hautes MM ap. absorbent plus l'UV que les fractions de faible MM ap. Ce résultat est en accord avec les travaux publiés par d'autres chercheurs (OLIVER et THURMAN, 1983).

Enfin si VAN BREEMEN et al. (1979) ont montré que la fraction de plus haute masse moléculaire des acides fulviques porte la majorité des fonctions carboxyles, la corrélation entre absorbance UV et les fonctions carboxyles ne peut pas être généralisée aux acides fulviques proprement dit (tableau 3).

Tableau 4 Absorbance UV rapportée à l'unité de concentration carbonée des différents uitrafiltrats des acides fulviques * Landes * (AFL), * Moulin Papon " (AFMP) et a Pinail " (AFP).

Table 4 Ultrafiltration of the "Landes" (AFL), "Moulin Papon" (AFMP) and * Pinail " (AFP) fulvic acids : UV absorbance (per $\mathrm{mg}^{-1} \mathrm{C}$ ) of the studied fractions.

\begin{tabular}{|llllll|}
\hline \multicolumn{5}{|c|}{ Absortance, $270 \mathrm{~nm}, 1 \mathrm{~cm}$ (rapportée au mg - $^{-1} \mathrm{C}$ ) } \\
\hline \multirow{2}{*}{ AFMP } & $\begin{array}{l}\text { Acide fulvique } \\
\text { non ultrafiltré }\end{array}$ & $\begin{array}{l}<30000 \\
\text { Daltons }\end{array}$ & $\begin{array}{c}<10000 \\
\text { Daltons }\end{array}$ & $\begin{array}{c}<1000 \\
\text { Daltons }\end{array}$ & $\begin{array}{l}<500 \\
\text { Daltons }\end{array}$ \\
\hline AFL & 0,022 & 0,021 & 0,014 & 0,011 & 0,011 \\
AFMP & 0,031 & 0,029 & 0,021 & 0,018 & 0,016 \\
AFP & 0,036 & 0,03 & 0,019 & 0,014 & 0,013 \\
\hline
\end{tabular}

\section{Réactivité avec le chlore}

Dans le but didentifier les principaux sous-produits de chloration des acides fulviques aquatiques, une solution concentrée $\left(1 \mathrm{gl}^{-1}\right)$ d'acide fulvique « Pinail * a été chlorée dans des conditions semblables à celles utilisées pour l'analyse des potentiels de réactivité (cł. techniques analytiques), puis acidifiée et extraite par l'éther éthylique. La figure 8 représente le chromatogramme reconstitué de l'analyse par chromatographie gazeuse 


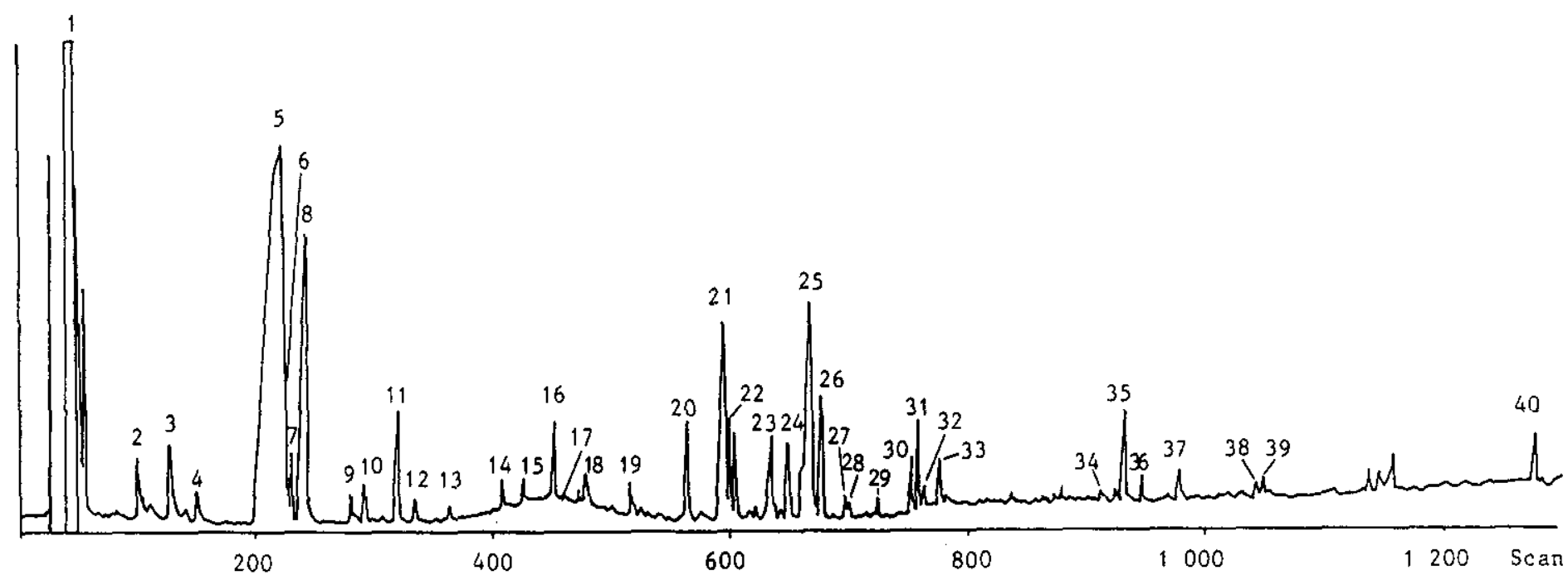

d'une solution de l'acide fulvique * Pinail ";
AFP $=1 \mathrm{~g}^{-1} ; 3,6 \mathrm{mg} \mathrm{Cl} / \mathrm{mg} \mathrm{C} ; \mathrm{pH} \approx 7,5 ; \mu=10^{-1} \mathrm{M}$ (phosphates); $72 \mathrm{hrs}$.
Colonne $=\mathrm{CP}$ Wax $51\left(50^{2} \mathrm{~m}\right) ;$ four $=50^{\circ} \mathrm{C}(8 \mathrm{~min}), 2^{\circ} \mathrm{C} / \mathrm{min}, 220^{\circ} \mathrm{C}$ (32 min).

Chlorination of "Pinail " fulvic acid (AFP) : GC/MS analysis of methylated ether extract.

$A F P=1 \mathrm{gt}^{-1} ; 3,6 \mathrm{mg} \mathrm{Cl} / \mathrm{mg} \mathrm{C} ; \rho H=7.5 ; \mu=10^{-1} \mathrm{M}$ (phosphate buffer); $72 \mathrm{hrs}$.

GC conditions: column $=C_{P}$ Wax $51(50 \mathrm{~m}) ;$ oven $=50^{\circ} \mathrm{C}(8 \mathrm{~min}), 2^{\circ} \mathrm{C} / \mathrm{min}, 220^{\circ} \mathrm{C}(32 \mathrm{~min})$. 
couplée à la spectrométrie de masse (impact électronique) de l'extrait méthylé au diazométhane. Les produits identifiés à partir des spectres obtenus par impact électronique et ionisation chimique sont rassemblés dans le tableau 5 sous trois rubriques :

- composés dont la structure proposée a été confirmée en comparant leur spectre de masse à ceux d'une banque de données,

- composés dont la structure proposée est probable,

- composés dont la structure présentée est incertaine ou incomplète.

Les composés identifiés sont essentiellement des esters méthyliques ou éthyliques; l'estérification est probablement due, dans tous les cas, à l'action du $\mathrm{CH}_{2} \mathrm{~N}_{2}$ sur les acides correspondants. Parmi ces composés, les acides aliphatiques chlorés à courte chaîne $\left(\mathrm{C}_{2}\right.$ à $\left.\mathrm{C}_{4}\right)$ sont les plus nombreux et les plus importants si on se réfère à l'inteinsité de leur pic chromatographique. Outre les acides dichloroacétique et trichloroacétique, on peut citer l'acide dichloromalonique, les acides chloromaléique et dichloromaléique, les acides chlorosuccinique et dichlorosuccinique.

Notons qu'aucun des composés chlorés identifiés n'est présent dans le chromatogramme obtenu à partir de l'échantillon d'acide fulvique non chloré. Par contre, certains composés non chlorés analysés figurent a la fois dans le chromatogramme de l'acide fulvique chloré et dans celui de l'acide fulvique non chloré (tableau 5).

Dans le but de quantifier plus précisément la formation de composés organo-chlorés, nous avons analysé le potentiel de formation (PF) de TOX et de THM en parallèle de la demande en chlore dans les mêmes conditions opératoires. Les résultats obtenus sont donnés dans le tableau 3 pour les onze acides fulviques extraits. Notons que les THM sont essentiellement représentés par le chloroforme, les organo-halogénés mixtes étant formés en quantités négligeables.

Les potentiels de réactivité avec le chlore sont très dépendants de la nature de l'acide fulvique (tableau 3), en accord avec RECKHOW (1984) qui signale des potentiels de demande en chlore variant de 0,55 à $0,8 \mathrm{mg} \mathrm{Cl} / 2 / \mathrm{mg} \mathrm{AF}$, des PFTHM de 15 à $25 \mu \mathrm{g} \mathrm{CHCl}_{3} / \mathrm{mg} \mathrm{AF}$ et des PFTOX de 70 à $120 \mu \mathrm{g} \mathrm{Cl} / \mathrm{mg} \mathrm{AF}$, pour 5 acides fulviques extraits d'eau de surface des USA et chlorés dans des conditions très voisines de celles utilisées dans notre étude. Le pourcentage de PFTOX attribué au PFTHM est d'après nos travaux compris entre 20 et $33 \%$, d'après RECKHOW (1984) de 18 à $21 \%$ pour 5 acides fulviques aquatiques et selon NORWOOD et al. (1985) de 17 à $33 \%$ pour 9 eaux de surface des USA.

Une autre part importante du PFTOX des acides fulviques peut être attribuée aux acides trichloro et dichloroacétiques, 11 à $19 \%$ et 5 à $6 \%$ respectivement, d'après RECKHOW (1984). Quelques analyses réalisées après chloration de trois des acides fulviques d'origine française ont permis de confirmer ces résultats, comme le montre le tableau 6. Le chloroforme, l'acide trichloroacétique et l'acide dichloroacétique peuvent donc représenter jusqu'à la moitié des composés organo-chlorés totaux formés lors de la chloration d'acide fulvique. 
Tableau 5 Quelques produits de chloration (méthylés par $\mathrm{CH}_{2} \mathrm{~N}_{2}$ ) de l'acide fulvique "Pinail ", après analyse par CG/SM (fig. 8).

Table 5 "Pinall "fulvic acid chlorination by-products (CG/MS analysis of methylated ether extracted, figure 8).

\begin{tabular}{|c|c|c|c|}
\hline Structures confirntes & & Structures probebles & Structures incertaines ou incomplites \\
\hline 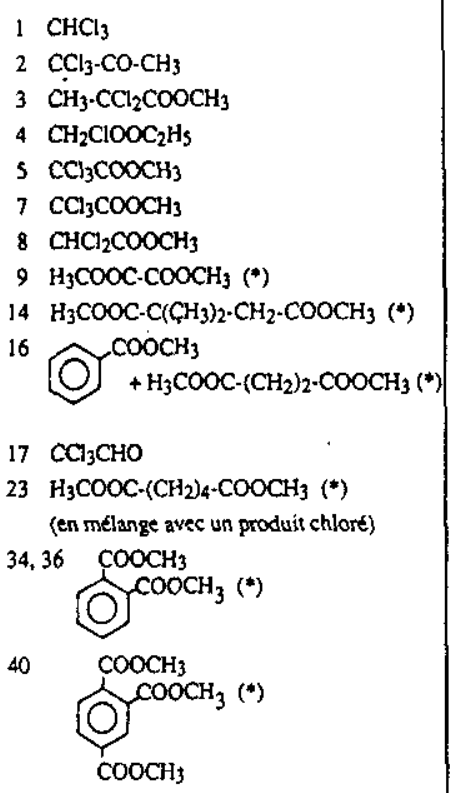 & $\begin{array}{l}6,10,11 \\
12 \\
20,24 \\
21 \\
22 \\
25 \\
26,28\end{array}$ & 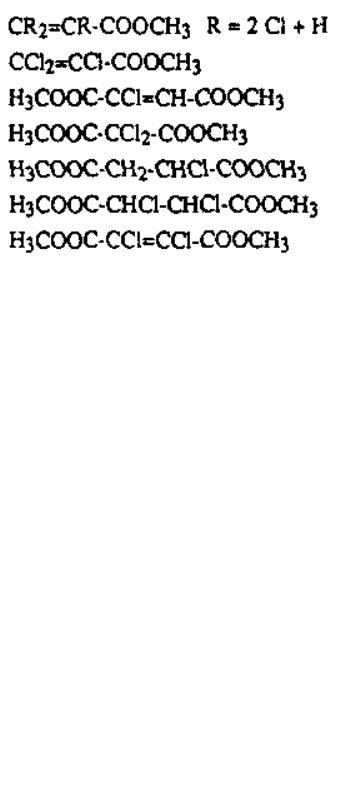 & 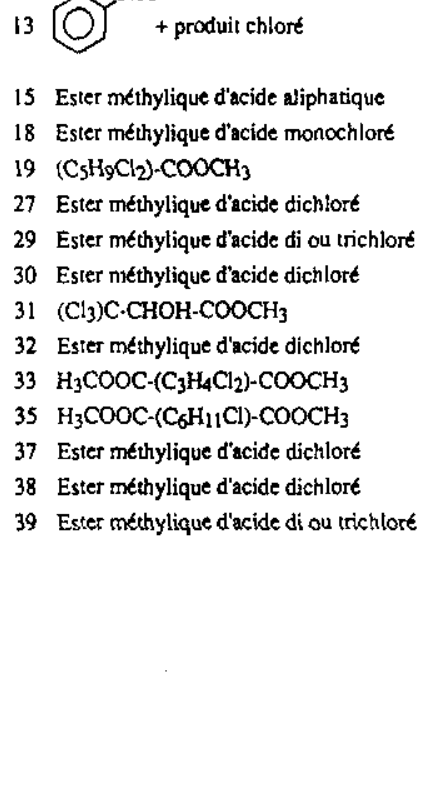 \\
\hline
\end{tabular}

(*) cornposes presents dans le chromatogramme de l'extrait de l'acide fulvique Pinail non chlore 
Tableau 6 Participation au TOX des sous-produits majoritaires de chloration de quelques acides fulviques.

Table 6 Contribution to the TOX formation of the major chlorination by-products of three studied fulvic acids.

\begin{tabular}{|lllll|}
\hline Aclde fulvique & $\begin{array}{l}\mathrm{CHCl}_{3} / 70 \mathrm{OX} \\
(\% \mathrm{Cl} / \mathrm{Cl})\end{array}$ & $\begin{array}{l}\mathrm{CCl}_{3} \mathrm{COOH} / \mathrm{TOX} \\
(\% \mathrm{CVCl})\end{array}$ & $\begin{array}{l}\mathrm{CHCl}_{2} \mathrm{COOH} / \mathrm{TOX} \\
(\% \mathrm{Cl} / \mathrm{Cl})\end{array}$ & $\begin{array}{l}\mathbf{5} \mathrm{TOX} \\
(\% \mathrm{Cl} / \mathrm{Cl})\end{array}$ \\
\hline AFL & 21,1 & 11,6 & 5,3 & 38,0 \\
AFMP & 31,6 & 23,5 & 10,3 & 65,4 \\
AFP & 30,1 & 16,6 & 6,3 & 53,0 \\
\hline
\end{tabular}

La figure 9 montre qu'il existe une corrélation entre l'absorbance UV et les potentiels de réactivités avec le chlore. II n'est pas surprenant que l'absorbance UV qui est principalement fonction du caractère aromatique des acides fulviques soit proportionnelle aux potentiels de réactivité avec le chlore, puisque le chlore est un réactif électrophile (MORRIS, 1978; CARLSON et CAPLE, 1978 ; MERLET, 1986 ; DE LAAT et al., 1982) agissant principalement sur les sites insaturés des composés organiques. Des études réalisées sur des composés simples et a pH neutre ont montré que les produits les plus réactifs avec le chlore sont généralement les aromatiques simples et polynucléaires substitués par un ou plusieurs groupements activants et à moindre effet les amines, les acides aminés et les cétones (B-dicétones) (DE LAAT et al., 1982 ; RECKHOW, 1984).

Ce phénomène a déjà été observé par d'autres auteurs qui signalent que la formation de chloroforme augmente avec la masse moléculaire apparente des substances humiques (OLIVER et VISSER, 1980 ; JOYCE ot UDEN, 1984 ; RECKHOW, 1984) donc avec l'absorbance UV. II en est de même avec l'augmentation de la couleur (OLIVER et THURMAN, 1983).

\section{CONCLUSIONS}

1. La méthode d'extraction des substances humiques aquatiques mettant en œuvre une adsorption sur résine macroporeuse XAD-8 à pH acide, ne permet de récupérer que $15 \%$ à $35 \%$ de la matière organique dissoute (exprimée en COD), dont la plus grande partie est représentée par la fraction fulvique plus soluble (60 à $90 \%$ massique).

2. Les acides fulviques extraits d'eaux de surface françaises contiennent en moyenne $51,5 \%$ de carbone et $1,4 \%$ d'azote dans leur structure tandis que les acides humiques présentent une teneur en azote significativement plus élevée. Les acides fulviques absorbent en UV sans présenter de bandes caractéristiques, leur absorbance à $254 \mathrm{~nm}$ rapportée à l'unité de concentration $\mathrm{mg} \mathrm{F}^{-1}$ de carbone varie de 0,025 a $0,040 \mathrm{uDO} / \mathrm{mg} \mathrm{C}^{-1} \mathrm{~cm}$, selon l'origine. En outre, ces composés comportent des quantités importantes de 

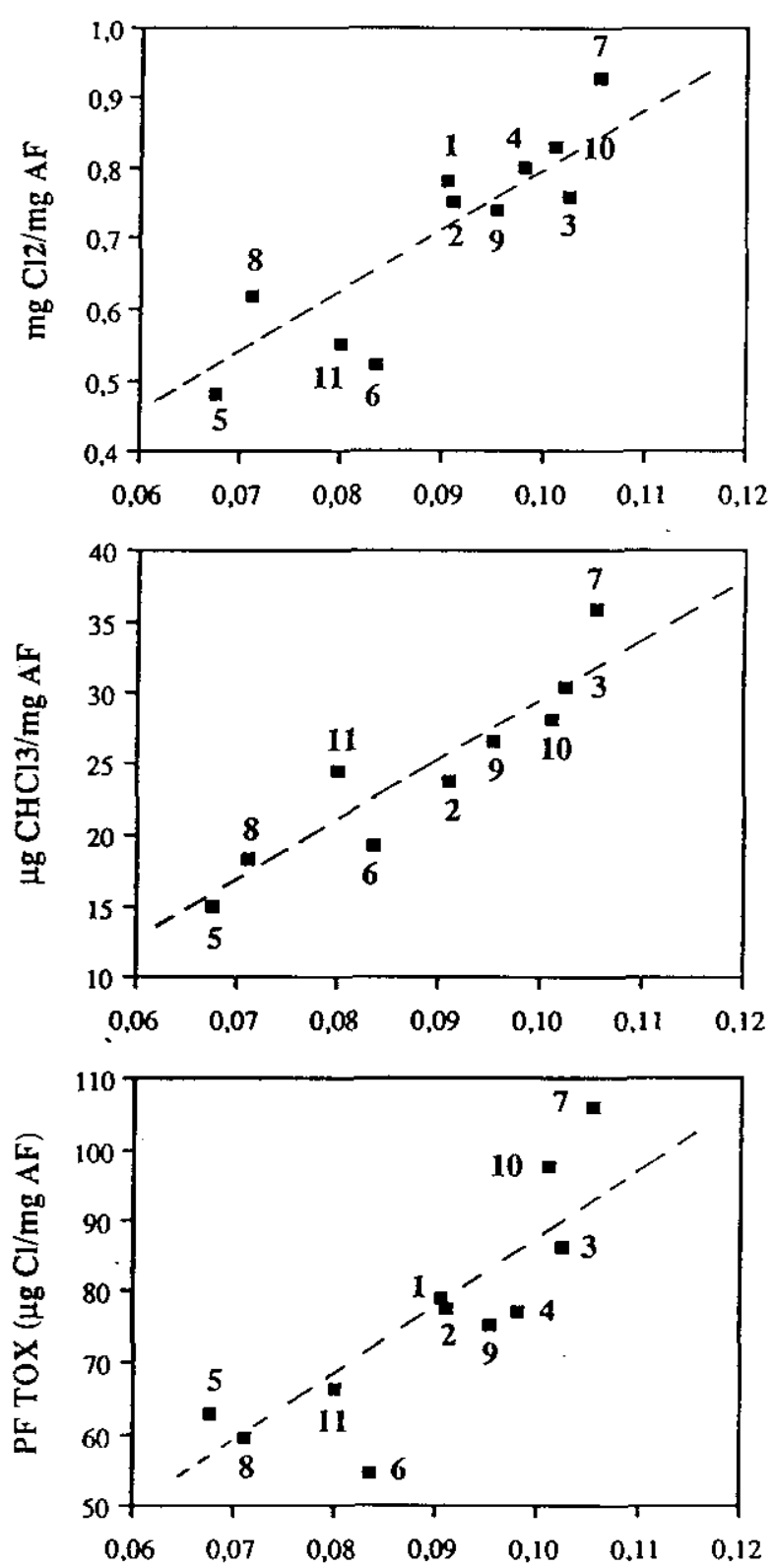

Abs. $254 \mathrm{~nm}$ - $5 \mathrm{~cm}$ (rapportée au mg /-1 AF)

Figure 9 Corrélation entre l'absorbance UV $(254 \mathrm{~nm}, 5 \mathrm{~cm})$ et les potentiels de réactivité avec le chlore, pour les acides fulviques étudiés.

(1: AFB ; 2 : AFC; 3 : AFG ; $4:$ AFGat ; $5:$ AFL ; $6:$ AFMP ; 7 : AFP ; 8 : AFSe ; $9:$ AFSO ; $10:$ AFV ; $11:$ AFVj).

Correlation between UV absorbance $(254 \mathrm{~nm}, 5 \mathrm{~cm})$ and the chlorine reactivity potentials of the studied fulvic acids.

(1: AFB ; $2:$ AFC ; $3:$ AFG ; $4:$ AFGat ; $5:$ AFL ; $6:$ AFMP ; 7 : AFP ; $8:$ AFSe ; $9:$ AFSO ; $10:$ AFV; $11:$ AFV $)$. 
fonctions carboxyles dans leur structure (moyenne de $12 \mu \mathrm{eg} / \mathrm{mg} \mathrm{C}$ ) correspondant approximativement à 1 atome de carbone sur 7 , inclus dans une fonction carboxyle.

3. Les acides fulviques réagissent d'autant mieux avec le chlore que leur absorbance UV (à $254 \mathrm{~nm}$ ) est élevée, c'est-à-dire que leur masse moléculaire apparente est importante, comme le montrent les résultats d'ultrafiltration. Cette réactivité avec le chlore se traduit, d'une part, par un potentiel de consommation de chlore variant de 1 a $1,8 \mathrm{mg} \mathrm{Cl}_{2} / \mathrm{mg} \mathrm{C}$ selon l'origine de l'acide fulvique, et d'autre part, par la formation de nombreux produits organochlorés (identifiés par CG/SM) dont les principaux sont le chloroforme, l'acide trichloroacétique et l'acide dichloroacétique. Enfin, notons que les potentiels de formation de chloroforme et de composés organohalogénés totaux varient de 30 à $70 \mu \mathrm{g} \mathrm{CHCl} / 3 \mathrm{mg} \mathrm{C}$ et de 100 à $200 \mu \mathrm{g}$ de $\mathrm{Cl} / \mathrm{mg} \mathrm{C}$, le potentiel de formation de chloroforme représentent en moyenne $29 \%$ du potentiel de formation de composés organohalogénés totaux.

\section{REMERCIEMENTS}

Les auteurs tiennent à remercier les directions et les personnels des usines d'eau potable de la Compagnie Générale des Eaux (Bellac, La Rochesur-Yon, Montceau-les-Mines, Rennes) et de la Compagnie des Eaux et de I'Ozone (Chatellerault, Pornic, St Malo) ainsi que le CEMAGref de Gazinet (Cestas) pour l'approvisionnement en eaux brutes. Les auteurs remercient également le personnel d'ANJOU-RECHERCHE pour sa participation à l'extraction des substances humiques * Seine * et Damien SIGURET qui a effectué la majorité des manipulations lors de l'extraction des autres substances humiques.

\section{RÉFÉRENCES BIBLIOGRAPHIQUES}

AIKEN G.R., 1984. Evaluation of ultrafiltration for determining molecular weight of fulvic acid. Environ. Sci. Technol., 18 : 978-981.

AIKEN G.R., 1988. A critical evaluation of the use of macroporous resins for the isolation of aquatic humic substances. In : Humic substances and their role in the environment. F.H. Frimmel, R.F. Christman Ed., Dahlem Workshop Reports, John Wifley and Sons, 133-148.
BABCOCK D.B., SINGER P.C., 1979. Chlorination and coagulation of humic and fulvic acids. J. Am. Water Works Ass., 71 : 149 152.

BEN AMOR H., 1988. Le bioxyde de chlore dans la filiere de production des eaux de consommation : contribution à l'étude de son mécanisme d'action sur des molécules modèles et sur les substances humiques 
aquatiques, Doctorat de l'Université de Poitiers, $n^{\circ}$ d'ordre : 175.

BRUCHET A., Nov. 1985. Applications de la technique de pyrolyse CG-SM à l'étude des matières organiques non volatiles des eaux naturelles ou en cours de traitement. Doctorat de $3 e$ Cycle, Universite de Poitiers, $n^{\circ} 1050$.

CARLSON R.M., CAPLE R., 1978. Organochemical implications of water chlorination. In: Water Chlorination Environmental Impact and Health Effects. R.L. Jolley Ed. (Ann Arbor Science Publishers), vol. 1. 65-75.

CHRISTMAN F.R., NORWOOD D.L., SEO Y., FRIMMEL F.H., 1987. Oxidative degradation of humic substances from freshwater environments. In : Humic substances $11:$ In Search of structure. New York : John Wiley and Sons.

COLEMAN W.E., MUNCH J.N., KAYLOR W.H., STREICHER R.P., RINGHAND H.P., MEIER J.R., 1984. Gas chromatography/ Mass spectroscopy analysis of mutagene extracts of aqueous chlorinated humic acid. A comparison of the byproducts to drinking water contaminants. Environ. Sci. Technol., 18: 674-681.

COLLINS M.R., AMY G.L., STEELINK C., 1986. Molecular weight distribution, carboxylic acidity and humid substance content of aquatic organic matter: Implication for removal during water treatment. Environ. Sci. Technol., 20 : 1028-1032.

CROUE J.P., 1987. Contribution à rétude de l'oxydation par le chiore et fozone d'acides fulviques naturels extraits d'eaux de surface, Doctorat de I'Université de Poitiers, $n^{\circ}$ d'ordre : 89.

DE GREEF E., MORRIS J.C., VAN KREIJL C.F., MORRA C.F.H., 1980. Health effects in the chemical oxidation of polluted waters. In : Water Chlorination: Environmental Impact and Health Effects, R.L. Jolley Ed. (Ann Artor Science Publishers), vol. 3, 913-924.

DE LAAT J., 1988. Contribution à l'étude du mode d'élimination de molécules organiques modeles sur charbon actif en grains : interactions entre les processus d'adsorption et de biodégradation, Doctorat es Sciences de l'Université de Poitiers, $n^{\circ}$ d'ordre : 485 .
DE LAAT J., MERLET N., DORE M., 1982. Chloration de composés organiques: Demande en chlore et réactivité vis-à-vis de la formation des trihalométhanes. Incidence de l'azote ammoniacal, Wat. Res., 76 : 1437-1450.

EWALD M., BELIN C., BERGER P., WEBER J.H., 1983. Corrected fluorescence spectra of fulvic acids isolated from soil and water. Environ. Sci. Technol., 17: 501-504.

GJESSING E.T., LEE G.F., 1967. Fractionation of dissolved organic matter in natural waters on sephadex columns. Environ. Sci. Technol., $1: 631-638$

GUITTONNEAU S., 1986. Contribution a l'étude de l'oxydation par couplage UV. peroxyde d'hydrogène de micropolluants organiques rêfractaires à l'oxydation, DEA Université de Poitiers.

HEMMING J., HOLMBOM B., REUNANEN M., KRONBERG L., 1986. Determination of the strong mutagen 3-chloro -4 (dichtoromethyl) -5-hydroxy $-2(5 \mathrm{H})$ furanone in chlorinated drinking and humics waters. Chemos phere, $15: 549-556$.

JADAS-HECART A., VENTRESQUE C., LEGUBE B., DORE M., 1988. Effect of ozonation on the chlorine demand of a treated surface water and some macromolecu lar compounds. IOA London Conference : Ozone in water quality management, 19th and 20th October.

JOHNSON J.D., CHRISTMAN R.F., NORWOOD D.L., MILLINGTON D.S., 1982. Reaction products of aquatic humic substances with chlorine. Environ. Health Perspectives, $46: 63-71$.

JOYCE W.D.A., UDEN P.C., 1984. THM precursors in the environment, J. Am. Water Works Ass., 76 : 102-106.

KOOL H.J., VAN KREIJL C.F., HRUBEC J. 1984. Mutagenic and carcinogenic properties of drinking water. In : Water Chlorination : Chemistry, Environmental Impact and Health Effects, R.L. Jolley Ed. (Lewis publishers, INC), vol. 5, 187-228.

KRINGSTAD K.P., LJUNGQUIST P.O.; DE SOUSA F., STROMBERG L.M., 1983. On the formation of mutagens in the chlorination of humic acid. Environ. Sci. Technol., $17: 553-555$.

KRONBERG L., HOLMBOM B., TIKKANEN L., 1987. Identification of the strong mutagen 
3-chloro -4-(dichloromethyl) -5-hydroxy $-2(5 \mathrm{H})$ furanone and of its geometric isomer E-2-chloro -3-(dichloromethyl) -4 oxobutenoix acid in mutagenic fraction of chlorine treated humic water and in drinking waters. Sixth Conference on Water Chlorination, Oak Ridge, Tennessee, May 3-4.

LEENHER J.A., 1981. Comprehensive approach to preparative isolation and fractionation of dissolved organic carbon from natural waters and wastewaters. Envir. Sci. Technol., $15: 578-587$.

LEFEBVRE E., LEGUBE B., 1989. Coagulation par $\mathrm{Fe}$ (III) de substances humiques extraites d'eaux de surface : effet du pH et de la concentration en substances humiques. A paraître dans Water Research.

LEGUBE B., CROUE J.P., DE LAAT J., DORE M., 1987. Ozonation of an extracted aquatic fulvic acid : Theoretical and practical aspects, 8th Ozone World Congress, Zurich (Switzerland), September 15-18, Proceedings Volume 2 (Edited by International Ozone Association), pp. E.56-E.79.

LEGUBE B., CROUE J.P., RECKHOW D.A., DORE M., 1985. Ozonation of organic precursors : Effects of bicarbonate and bromide. Proceedings of the International Conference: The role of ozone in water and wastewater treatment. November 13-14. (Edited by Perry R. and Mc Intyre A.E.), Selper Ltd., London : 76-86.

LEGUBE B., LEFEBVRE E., BARBIER J., PAILLARD H., BOURBIGOT M.M., 1988. Removal of humic substances and manganese in slightly mineralized water by means of iron salts and ozone, AWWA Annual Conference \$ Exposition, Orlando (Florida), June 19-23.

LLAO W., CHRISTMAN R.F., JOHNSON J.D., MILLINGTON D.S., HASS J.R., 182. Structural characterization of aquatic humic material. Environ. Sci. Technol., 16: 403-410.

MAC CARTHY P., MALCOLM R.L., HAYES M.H.B., SWIFT R.S., SCHNITZER M., CAMPBEL W.L., 1986. Establishment of a collection of standard humic substances. IHSS 3rd International Meeting. August 4-8, Oslo (Nonway).

MALCOLM R.L., 1985. The geochemistry of stream fulvic and humic substances. Papport interne de U.S. Geological Survey Box 25046, Mail Stop 408, Federal Center, Denver, Colorado 80225.
MALCOLM R.L., MAC CARTY P., 1986, Limitations in the Use of Commercial Humic Acids in Water and Soil Research. Environ. Scl. Technol., 20 : 904-911.

MEIER J.R., RINGHAND H.P., COLEMAND W.E., MUNCH J.W., STREICHER R.P.. KAYLOR W.H., SCHENCK K.M., 1985, Identification of mutagenic compounds formed during chlorination of humic acid. Mutation Research, 157: 11-112.

MERLET N., 1986. Contribution à l'étưoe du mécanisme de formation des trihalométhanes et des composés organohalogénés non volatils lors de la chloration de molécules simples. Doctorat ès Sciences Physiques, Université de Poitiers, $n^{\circ} 426$.

MILLER J.W., UDEN P.C., 1983. Characterization of non volatile aqueous chlorination products of humic substances. Environ. Sci. Tochnol., 17: 150-157.

MORRIS J.C., 1978. The chemistry of aqueous chlorine in relation to water chlorination. In : Water Chlorination Environmental Impact and Health Effects. R.L. Jolley Ed. (Ann Arbor Science Publishers), vol. 1, 21-35.

NOACK M.G., DOERR R.L., 1978. Reaction of Chlorine, Chlorine Dioxide and Mixture There of with Humic Acid: An Interim Report. In : Water Chlorination : Environmental Impact and Health Effects, R.L. Jolley Ed. (Ann Arbor Science Publishers), vol. 2, 49-58.

NORWOOD D.L., JOHNSON J.D., CHRISTMAN R.F., MILUNGTON D.S., 1983. Chlorination products from aquatic humic material at neutral pH. In : Water Chlorination Environmental Impact and Health Effects, R.L. Jolley Ed. (Ann Arbor Science Publishers), vol. 4, 191-200.

NORWOOD D.L., THOMSON G.P., St AUBIN J.J., MILLINGTON D.S., CHRISTMAN R.F., JOHNSON J.D., 1985. Byproducts of chlorination: Specific compounds and their relationship to total organic halogen. Michigan, Lewis Publ., INC/Drinking Water Res. Found. (Réf. AFEE. 66/47895 - $n^{\circ} 352$ - 1985), 109 121.

NORWOOD D.L., 1988. Critical comparison of structural implications from degradative and non degradative approaches. In : Humic substances and their role in the environ- 
ment, F.H. Frimmel, R.F. Christman Ed., Dahlem Workshop Reports, John Willey and Sons, 133-148.

OGURA N., 1974. Molecular weight fractionation of dissolved organic matter in coastal seawater by ultrafiltration. Marine Biology : 24, 305-312.

OLIVER B.G., VISSER S.A., 1980. Chioroform production from the chlorination of aquatic humic material : the effect of molecular weight, environment and season. Wat. Res., 14: 1137-1141.

OLIVIER B.G., THURMAN E.M., 1983. Influence of aquatic humic substance properties on trihalomethane potential. In : Water Chorination : Environmental Impact and Health Effects, R.L. Jolley Ed. (Ann Arbor Science Publishers), vol. 4, 237241.

PAILLARD H., BRUNET R., DORE M., 1988. Optimal conditions for applying an ozonehydrogen-peroxide oxidizing system, Wat. Res., 22 : 91-103.

QUALLS R.G., JOHNSON J.D., 1983. Kinetics of the Short-Term Consumption of Chlorine by Fulvic Acid. Environ. Sci. Technol., 17: 692-698.

RECKHOW D.A., 1984. Organic halide formation and the use of pre-ozonation and alum coagulation to control organic halide precursors. Ph. D. Thesis, Department of Environmental Sciences and Engineering, Chapel Hill (N.C.).

RECKHOW D.A., LEGUBE B., SINGER P.C., (1986). The ozonation of organic halide precursors : Effect of bicarbonate. Wat. Res., 20 : 987-988.

RODIER J., 1984. L'analyse de l'eau : eaux naturelles, eaux résiduaires, eau de mer, 7e édition, Dunod Ed.
SCHNITZER M., KHAN S.U., 1972. Humic substances in the environment, Marcel Dekker, Inc., New York.

SCHULTEN H.R., ABBT-BRAUM G., FRIM.MEL F.H., 1987. Time-resolved pyrolysis field ionisation mass spectrometry of humic material isolated from freshwater. Environ. Sci. Technol., 21, 349-357.

SEEGER R., MOORE L.A., STEVENS A.A., 1984. Formation of acidic trace organic byproducts from chlorination of humic acids. in: Water Chlorination: Chemistry, Environmental Impact and Health Effects. R.L. Jolley Ed. (Ann Arbor Science Publishers, INC), vol. 5, 859-873.

THURMAN E.M., 1985. Developments in Biochemistry : Organic Geochemistry of Natural Waters. M. Nijhoff, Dr W. Junk Publishers, Dordrecht.

THURMAN E.M., MALCOLM R.L., 1981. Preparative isolation of aquatic humic substances. Environ. Sci. Technol., 15 : 463-466.

THURMAN E.M., MALCOLM R.L., 1983. Structural study of humic substances : New approaches and methods. In : Aquatic and Terrestrial Humic Materials, Christman R.F. and Gjessing E.T. Ed. (Ann Artor Science Publishers), 1-25.

VAN BREEMEN A.N., NIEUWSTAD T.J., VAN DER MEENT-OLIEMAN, 1979. The fate of fulvic acids during water treatment. Wat. Res., $13, n^{\circ} 8: 771-780$.

VISSER S.A., 1983. Fluorescence phenomena of humic matter of aquatic origin and microbial cultures. In : Aquatic and Terrestrial Humic Matrials, Christman R.F. and Gjessing E.T. Ed. (Ann Arbor Science Publishers), 183-202.

VOGEL A.T., 1956. A Text-Book of Practical Organic Chemistry. Third Edition, Longmans, 971-972. 